\title{
含 $\mathrm{CF}_{3} \mathrm{CF}_{2} \mathrm{CF}_{2} \mathrm{C}\left(\mathrm{CF}_{3}\right)_{2}$ 基团支链型氟表面活性剂的合成及性能研究
}

\author{
沙敏 ${ }^{a, b} \quad$ 张丁 $b$ 潘仁明 $b \quad$ 邢萍 ${ }^{a}$ 姜标*, $a, b$ \\ ( ${ }^{a}$ 中国科学院上海有机化学研究所 天然产物有机合成化学重点实验室 上海市徐汇区零陵路 345 号 200032) \\ ( ${ }^{b}$ 南京理工大学 化工学院 江苏省南京市玄武区孝陵卫 200 号 210094)
}

\begin{abstract}
摘要 全氟辛酸/全氟辛基磺酸(PFOA/PFOS)类氟表面活性剂因不易被生物降解且对环境有毒害作用, 被列为持久性有 机污染物. 采用引入氟碳支链的策略作为 PFOA/PFOS 替代物的研发取向, 以六氟丙烯二聚体为原料合成了新型阳离子 型、两性型、双子型和非离子型氟表面活性剂, 并对它们的表面活性和急性毒性性能进行了测试. 结果表明, 所合成的 支链型表面活性剂表面活性高且毒性低. 因此, 基于六氟丙烯二聚体(HFPD)合成 PFOS/PFOA 替代物是一种简单、经济 且环保的方法.
\end{abstract}

关键词 全氟辛酸(PFOA); 全氟-2-甲基-2-戊烯; 支链型氟表面活性剂; 表面张力; 临界胶束浓度; 急性毒性

\section{Synthesis and Properties Study of Novel Branched Fluorinated Surfactants with $\mathrm{CF}_{3} \mathrm{CF}_{2} \mathrm{CF}_{2} \mathrm{C}\left(\mathrm{CF}_{3}\right)_{2}$ Group}

\author{
Sha, Min $^{a, b} \quad$ Zhang, Ding $^{b} \quad$ Pan, Renming $^{b} \quad$ Xing, Ping ${ }^{a} \quad$ Jiang, Biao ${ }^{*, a, b}$ \\ ( ${ }^{a}$ CAS Key Laboratory of Synthetic Chemistry of Natural Substances, Shanghai Institute of Organic Chemistry, Chinese \\ Academy of Sciences, 345 Lingling Road, Shanghai 200032) \\ ( ${ }^{b}$ School of Chemical Engineering, Nanjing University of Science and Technology, 200 Xiaolingwei, Nanjing 210094)
}

\begin{abstract}
Perfluorooctanoic acid (PFOA) and perfluorooctane sulphonate (PFOS) were listed under Stockholm convention on persistent organic pollutants owing to their poor biodegradability and toxic effects in the environment, so various strategies were reported for synthesizing non-bioaccumulable alternatives to them. Herein, we described our recent strategies for synthesis of non-bioaccumulable fluorinated surfactants by introducing branched perfluorinated chain using perfluoro-2-methyl-2-pentene as starting material. Novel branched fluorinated cationic, gemini and amphoteric surfactants were designed and prepared through a four-step route. They all have low solubility in water and exhibit poor ability to reduce the surface tension of water. A series of branched fluorinated nonionic surfactants with different length of hydrophilic chains were synthesized via a three-step route. The solubility becomes larger with the increase of the value of polymerization degree of polyethylene glycol. But, the surface tension measurement results show that their ability and efficiency to reduce the surface tension of water change little when the value of polymerization degree of polyethylene glycol is equal to or greater than 4. All the values of surface activities of the novel $\mathbf{2 0} \sim \mathbf{2 2}$ surfactants (take the compound $\mathbf{2 0}$ for example, about 21.4 $\mathrm{mN} / \mathrm{m}$ at the $\mathrm{cmc} 4.4 \times 10^{-5} \mathrm{~mol} / \mathrm{L}$ ) are lower than that of sodium perfluorooctanoate (about $24.7 \mathrm{mN} / \mathrm{m}$ at the $\mathrm{cmc} 3.1 \times 10^{-2}$ mol/L). Methyl-benzenesulfonic acid (compound 23) was synthesized through a two-step route. Both the steps are easy work-up, mild reaction conditions, low cost and high yields. The surfactant exhibits excellent ability to reduce the surface tension of water to $20.8 \mathrm{mN} / \mathrm{m}$. Besides, it has good salt-resistance. The acute toxicity of $\mathbf{2 0}$ was investigated and the result shows that the acute oral toxicity $\mathrm{LD}_{50}$ Value of the compound 20 was $3160 \mathrm{mg} / \mathrm{kg}$ which is higher than that of ammonium perfluorooctanoate $\left(\mathrm{C}_{7} \mathrm{~F}_{15} \mathrm{COONH}_{4}, 470 \sim 540 \mathrm{mg} / \mathrm{kg}\right)$. So preparing branched fluorinated surfactants using hexafluoropropylene (HFPD) is a simple, economical and environmentally friendly method for synthesis of alternatives to PFOA and PFOS.
\end{abstract}

Keywords perfluorooctanoic acid (PFOA); perfluoro-2-methyl-2-pentene; branched fluorinated surfactant; surface tension; critical micelle concentration; acute toxicity

\section{1 引言}

与普通表面活性剂相比，氟表面活性剂具有高表面 活性的特性 ${ }^{[13]}$. 此外, 其还具有高耐热稳定性及高化 学稳定性. 氟表面活性剂通常由全碳氟链或部分氟代碳 氟链和亲水基团组成 ${ }^{[4 ~ 6]}$, 如最知名的有 PFOA (per- fluorooctanoic acid, $\mathrm{C}_{7} \mathrm{~F}_{15} \mathrm{CO}_{2} \mathrm{H}$ ) 和 PFOS (perfluorooctane sulphonate, $\mathrm{C}_{8} \mathrm{~F}_{17} \mathrm{SO}_{3} \mathrm{X}$, 其中, $\mathrm{X}$ 为 $\mathrm{K}, \mathrm{Na}$ 或 $\mathrm{H}$ ). 尽 管 PFOS/PFOA 类物质有众多的优点并被广泛使用，其 却因生物可降解性差且对环境有毒害作用而被斯德哥

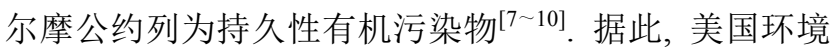
保护署(EPA)发布了自愿性 2010～2015 年全氟辛酸及其

*E-mail: jiangb@sioc.ac.cn; Tel.: 021-54925566; Fax: 021-64166128

Received March 13, 2015; published April 8, 2015.

Supporting information for this article is available free of charge via the Internet at http://sioc-journal.cn. 
盐类环境计划, 即 2010/2015 PFOA Stewardship Program; 该计划规定 PFOS/PFOA 类氟碳表面活性剂会被 逐步禁止生产、销售和使用 ${ }^{[11]}$. 因此, 研制全新的可降 解氟表面活性剂以全面取代现有的 PFOS/PFOA 类氟碳 表面活性剂, 并同时评价其生物降解性和对环境的影 响, 已成为紧迫的理论研究问题之一, 并引起了各国研 究人员的高度重视. 替代品的研发取向可大致分为三 类: 一, 降低全氟链的长度; 二, 氟碳链中引入 $\mathrm{N} 、 \mathrm{O}$ 等 杂原子及亚甲基或次甲基; 三, 引入氟碳支链. 替代品 的毒性和生物降解性的研究报导较少.

一、降低全氟链的长度. PFBS $\left(\mathrm{C}_{4} \mathrm{~F}_{9} \mathrm{SO}_{3} \mathrm{H}\right) 、 \mathrm{PFHS}$ $\left(\mathrm{C}_{6} \mathrm{~F}_{13} \mathrm{SO}_{3} \mathrm{H}\right)$ 以及由它们衍生而来的表面活性剂都被报 导. 研究表明, PFBS 无明显生物累积性, 可短时间内被 人体免疫系统除去, 且降解代谢产物无毒. 但是, 全氟 链长为 4 和 6 的表面活性剂的疏油性不及全氟链长为 8 的表面活性剂. 此外, 全氟链的制备较难, 通常用电解 氟化法 ${ }^{[12]}$ 和氟烯烃调聚法 ${ }^{[13 \sim 15]}$ 等合成而来. 电解氟化 法以 HF 为主要原料, 尽管 HF 比较廉价, 但是电解法会 产生很多副产物. 氟烯烃调聚法通常会生成不同链长的 混合物，因此必须严格控制反应条件.

二、氟碳链中引入 $\mathrm{N} 、 \mathrm{O}$ 等杂原子及亚甲基或次甲 基. 化合物中含有次甲基或亚甲基基团通常具有潜在的 可降解性, 基于这, 人们合成了一类含有 $\operatorname{VDF}($ 偏氟乙 烯)和 TFP(3,3,3-三氟丙烯) 低聚物链的表面活性剂来作 为 $\mathrm{PFOS} / \mathrm{PFOA}$ 的替代物 ${ }^{[16]}$. 该类方法大多采用氟烯烃 调聚法, 也面临着上述缺点.

三、引入氟碳支链. 研究表明, 直链的氟碳表面活 性剂在相对高的使用浓度下表现出最低的表面张力, 而 支链氟碳表面活性剂在相对低的浓度下使用, 降低表面
张力却更为有效. 例如, Dmowski 等 ${ }^{[17]}$ 合成了 $\mathrm{CF}_{3}$ $\mathrm{CF}_{2} \mathrm{CF}_{2} \mathrm{C}\left(\mathrm{CF}_{3}\right)_{2} \mathrm{CH}_{2} \mathrm{CH}_{2} \mathrm{COONa}$ ，并将其表面活性与 $\mathrm{CF}_{3}\left(\mathrm{CF}_{2}\right)_{6} \mathrm{COONa}$ 比较, 发现前者的性能优于后者. 氟 碳支链的制备方法主要是齐聚法, 最常见的有六氟丙烯 齐聚法. 六氟丙烯是仅次于四氟乙烯产量的简单氟化工 产品, 从成本的角度就适合于工业生产.

目前, 基于六氟丙烯齐聚物合成氟表面活性剂的研 究大多是基于 HFPT(六氟丙烯三聚体). 基于 HFPD(六

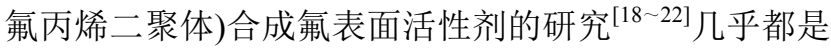
基于与 HFPD 中碳碳双键直接相连氟原子的活泼性. 对 基于 HFPD 中碳碳双键合成氟表面活性剂的研究极 少 ${ }^{[23 \sim 25]}$ 且均为国外报导，产品种类少(所报导的为羧酸 盐或磺酸盐阴离子型氟表面活性剂), 产品表面张力在 19 27 mN/m 间, 性能优良. 六氟丙烯二聚体总氟碳数 少(6 个)同时也不含有 $\mathrm{SO}_{2} \mathrm{~F}$ 基团, 对环境危害较低. 故 基于 HFPD 中碳碳双键合成支链型氟表面活性剂的方法 应受到重视.

针对目前氟碳表面活性剂的研发、生产和应用 ${ }^{[26-29]}$ 的不足, 我们课题组以 HFPD 为原料并以其碳碳双键为 反应活性位点成功开发了一系列氟表面活性剂 ${ }^{[30,31]}$ (图 式 1), 它们的性能均优于全氟辛酸钠. 对比化合物 $\mathbf{1} \sim \mathbf{2}$ 和基于 HFPT 开发的氟表面活性剂 $\mathrm{C}_{9} \mathrm{~F}_{17} \mathrm{OC}_{6} \mathrm{H}_{4} \mathrm{CONH}-$ $\mathrm{C}_{3} \mathrm{H}_{6} \mathrm{~N}^{+}\left(\mathrm{CH}_{3}\right)_{3}{ }^{-}{ }^{-}$的表面活性性能, 发现它们具有类似的 性能. 基于这些研究成果, 本文在前期工作的基础上, 依旧基于 HFPD 中碳碳双键的活泼性设计、合成其它氟 表面活性剂，测试各项性能，从中寻找结构和性能间的 关系，希望能为今后氟表面活性剂的开发和应用等研究 提供一定的实验和理论支撑.

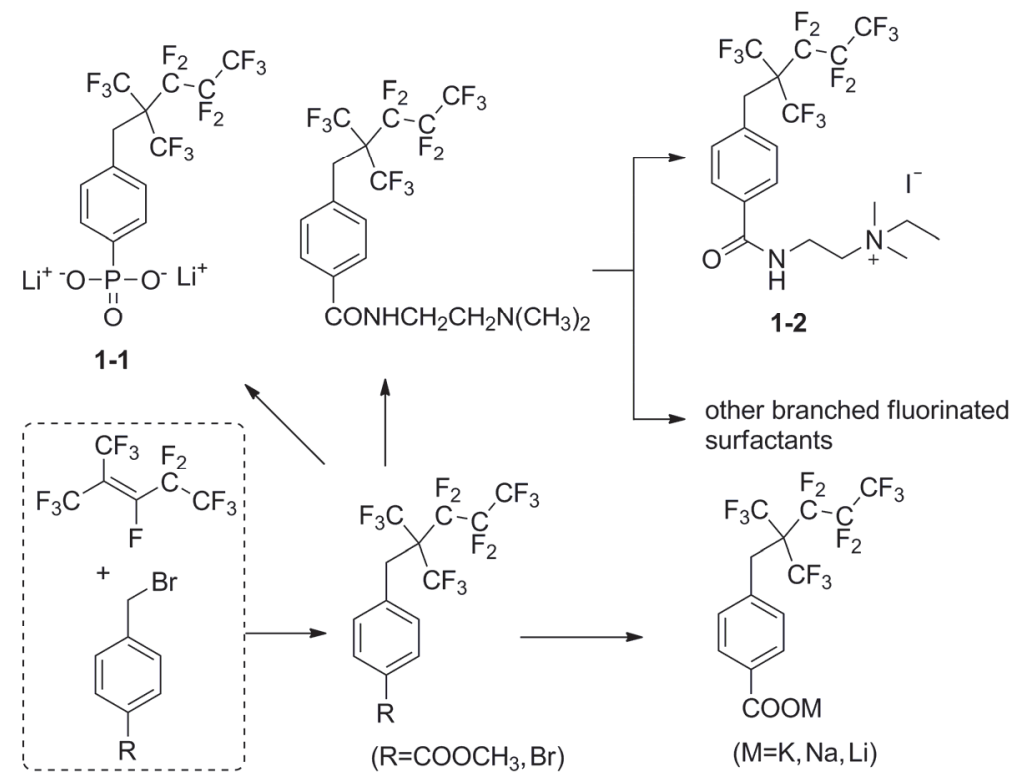

图式 1 基于 HFPD 的氟表面活性剂的合成路线

Scheme 1 Synthesis of fluorinated surfactants based on HFPD 


\section{2 结果与讨论}

\section{1 化合物合成讨论}

在前期工作中, 我们合成了如图式 1 所示的表面活 性剂，我们希望将图式 1 中的 $\mathrm{R}$ 换成 $\mathrm{SO}_{2} \mathrm{OCH}_{3}$ 来合成含 磺酰基的中间体化合物进而合成氟表面活性剂，但是， 经过大量的条件摸索(反应投料比、温度、反应时间、原 料投料顺序), 均得不到目标产物. 随后, 我们将 4-溴甲 基苯磺酸甲酯换成氯乙基磺酰氟来研究这类反应，也无 法得到目标化合物(如图式 2). 综上，通过对含有磺酰基 基团的底物发生亲核取代反应引入含支链的全氟基团 的方法不可行，因此，我们打算先引入全氟基团，再引 入磺酰基基团.
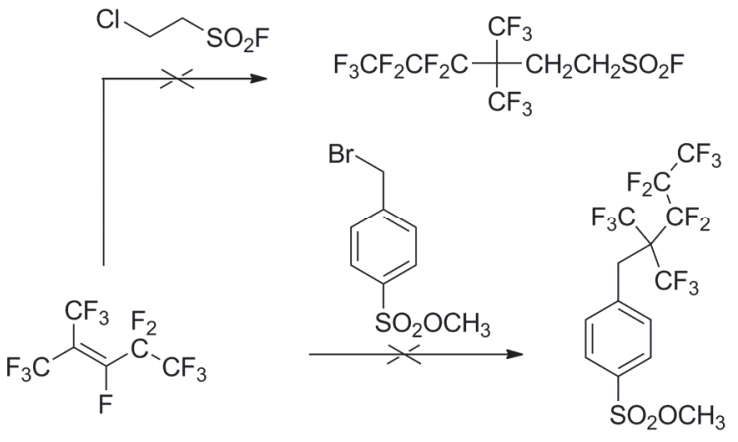

图式 2 底物含 $\mathrm{SO}_{2}$ 基团的亲核取代反应

Scheme 2 Nucleophilic substitution reaction with the substrate containing $\mathrm{SO}_{2}$ group

选择溴化苄为原料, 并设想按图式 3 中的路线经四 步合成氟表面活性剂. 对可能影响第二步反应的因素 (反应温度、氯磺酸的加入量以及滴加速度)进行摸索, 发现不管反应条件如何改变, 产物均为间位和对位取代 的混合物, 且很难通过常规的方法将两者大量分离. 因 此, 我们按照图式 4 的路线得到了三种表面活性剂的混
合物 A、B 和 C. 同时，通过过柱分离得到了少量中间体 化合物 2 和 3 的纯品，化合物 2 和 3 分别按图式 4 的合 成路线分别得到最终产物 6 11 的纯品, 用于定性分析. 经核磁 $\mathrm{H}$ 谱分析可得混合物中各物质的物质的量含量, 混合物 $\mathrm{A}$ 的组成为 $8: 9=59 \%: 41 \%$; 混合物 $\mathrm{B}$ 的组成 为 $10: 11=67 \%: 33 \%$; 混合物 $\mathrm{C}$ 的组成为 $6: 7=$ $65 \%: 35 \%$.

为了得到单一的产物，就要用苯环上间位或对位有 取代基的底物为原料. 溴化茮有两个间位位点，且间位 均有取代基的化合物不易获得、价格昂贵，因此使用苯 环上对位有取代基的底物. 考虑到取代基的种类对最终 产品的性能会有一定的影响, 且为了将产品性能与前面 的工作进行对比, 就要使取代基的影响最小化. 因为取 代基的位置在苯环上，属于疏水链部分，所以取代基也 应是疏水性的; 考虑到取代基的空间效应会影响分子的 柔顺性和水溶性，综上，我们选择体积较小的甲基为取 代基. 以 4-甲基溴化苄为起始原料, 按图式 5 的路线成 功合成了一系列氟表面活性剂, 所有的反应步骤操作简 单、条件温和. 中间体和最终产物的结构经 ${ }^{1} \mathrm{H} \mathrm{NMR},{ }^{19} \mathrm{~F}$ NMR, ${ }^{13} \mathrm{C}$ NMR, FTIR 或 MS 表征, 验证为目标化合物.

\section{2 表面张力测试结果与讨论}

采用德国 Krüss 公司产的 K100 表面张力测试仪，用 Wilhemy 板法测定溶液在 $25{ }^{\circ} \mathrm{C}$ 时的表面张力. 将 2.1 节中合成的氟表面活性剂配制成不同浓度水溶液后静 置过夜测定，测试时按照浓度由低到高的顺序，每种浓 度水溶液测量三次后取平均值, 以测得的表面张力数值 为纵坐标, 浓度为横坐标作图, 得到水溶液表面张力随 浓度变化曲线.

混合物 $\mathrm{A}$ 和 $\mathrm{B}$ 的水溶性较差, $\mathrm{C}$ 的水溶性较好, $\mathrm{A}$ 、 B、C 水溶液表面张力随浓度变化曲线如图 1. 在饱和溶 液浓度下，混合物 A 可将水溶液表面张力降至 25.8

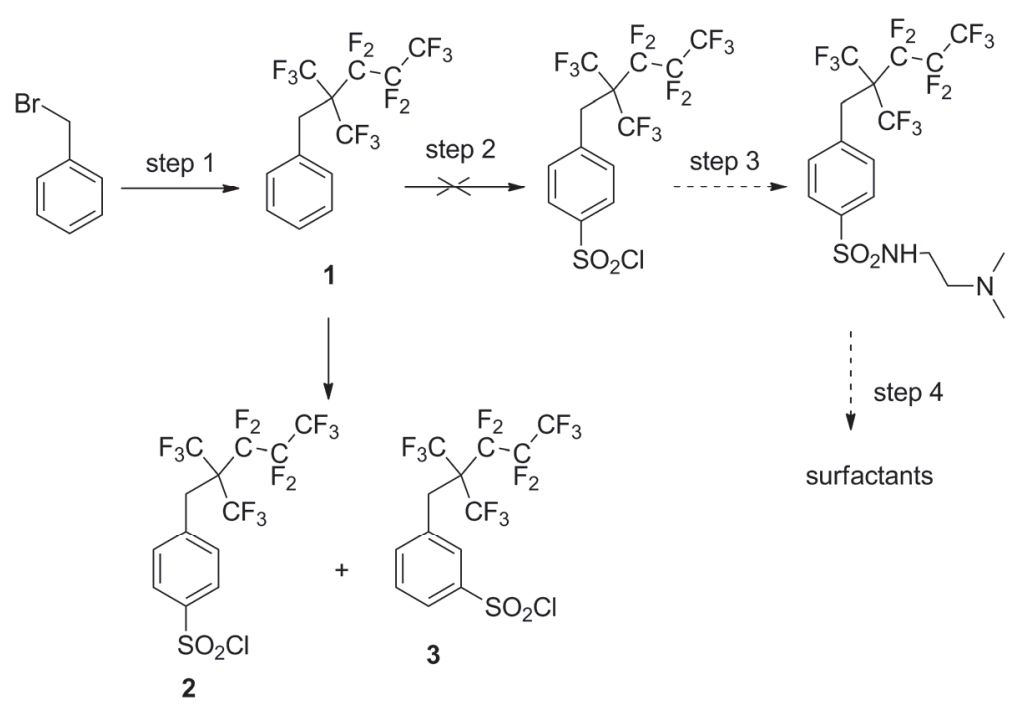

图式 3 以澳化芐为原料的表面活性剂的合成路线

Scheme 3 Synthetic route to produce surfactants with bromomethyl-benzene as starting material 


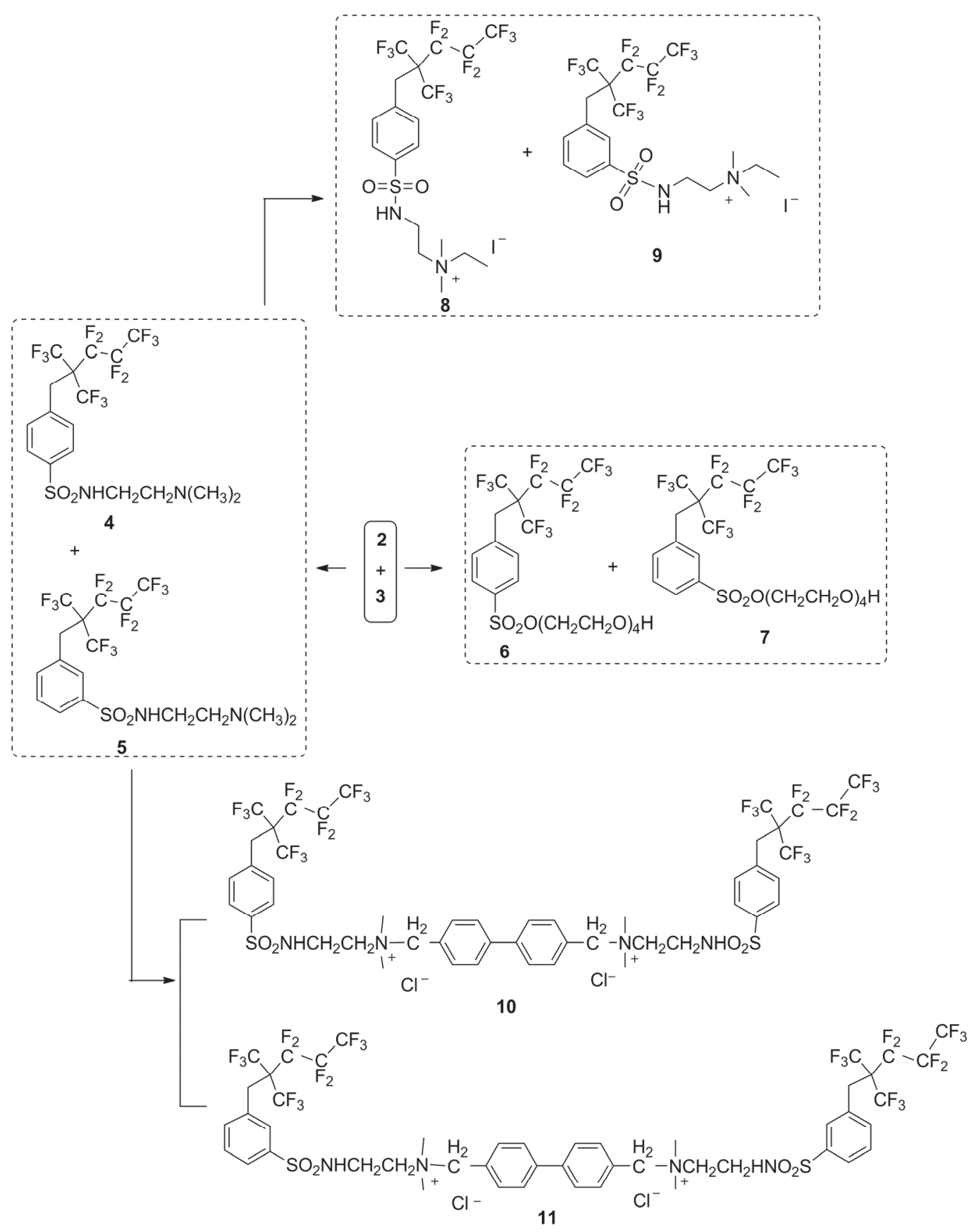

图式 4 三种氟表面活性剂的合成路线

Scheme 4 Synthetic route to produce three kinds of surfactants

$\mathrm{mN} / \mathrm{m}$, 混合物 B 仅将水溶液表面张力降至 $29.7 \mathrm{mN} / \mathrm{m}$. 混合物 $\mathrm{C}$ 显示出最强的降低表面张力的能力, 其可将水 溶液表面张力降至 $20.4 \mathrm{mN} / \mathrm{m}$.

化合物 15 17 的水溶性很差, 它们的饱和溶液浓 度均低于 $1.0 \times 10^{-3} \mathrm{~mol} / \mathrm{L}$, 它们的水溶液表面张力随浓 度变化的测试结果如图 2. 阳离子型表面活性剂(15)和 双子型表面活性剂(17)在饱和溶液浓度下可将水溶液表 面张力降至 $32.1 \mathrm{mN} / \mathrm{m}$, 而两性型表面活性剂(16)的表 面活性性能最差, 在饱和溶液浓度下仅将水溶液表面张 力降至 $38.9 \mathrm{mN} / \mathrm{m}$.
我们设计并成功合成了以聚乙二醇为亲水链的氟 表面活性剂，拟增加产物的水溶性，同时为了考察聚乙 二醇聚合度对表面活性剂表面活性的影响，合成了不同 聚合度的含全氟支链的非离子型氟表面活性剂 $\mathbf{1 8} \sim \mathbf{2 2}$. 化合物 $18 \sim 22$ 的水溶性大大优于 $15 \sim 17$, 且随着聚合 度的增加, 产物溶解度增大. 化合物 $18 \sim 22$ 的水溶液表 面张力随浓度变化的测试结果如图 3.

由图 3 知: 化合物 18 的临界胶束浓度为 $2.7 \times 10^{-4}$ $\mathrm{mol} / \mathrm{L}$, 临界胶束浓度下的表面张力值为 $24.8 \mathrm{mN} / \mathrm{m}$; 化 合物 19 的临界胶束浓度为 $1.5 \times 10^{-4} \mathrm{~mol} / \mathrm{L}$, 临界胶束 


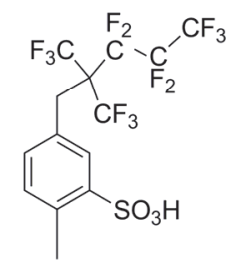

23

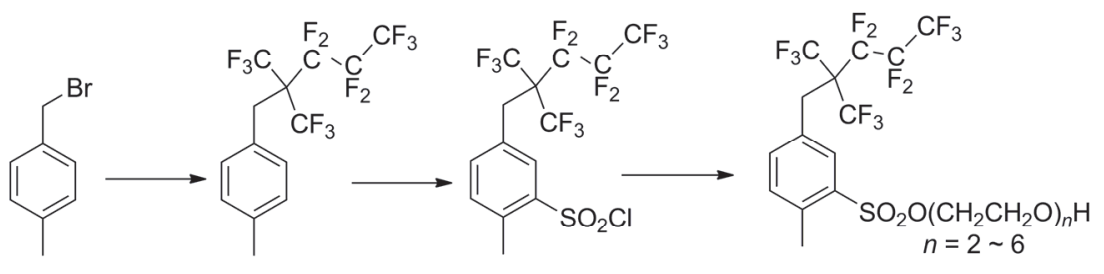

12

13

$18 \sim 22$<smiles>Cc1ccc(CC(F)(F)C(F)(F)CC(F)(F)F)cc1S(=O)(=O)NCCN(C)CC(=O)O</smiles>

16<smiles>CNCCNS(=O)(=O)c1cc(CC(F)(C(F)(F)F)C(F)(F)CC(F)(F)F)cc(C)c1C</smiles>

14<smiles>CCN(C)CCNS(=O)(=O)c1cc(CC(F)(F)C(F)(F)CCC(F)(F)F)ccc1C</smiles>

15<smiles>Cc1ccc(CC(C(F)(F)F)(C(F)(F)F)C(F)(F)F)cc1S(=O)(=O)NCCN(C)[I-]</smiles>

$\mathrm{Cl}^{-}$<smiles>Cc1ccc(-c2ccccc2)cc1</smiles>

17

图式 5 新型支链型氟表面活性剂的合成路线

Scheme 5 Synthetic route to produce the novel branched fluorinated surfactants

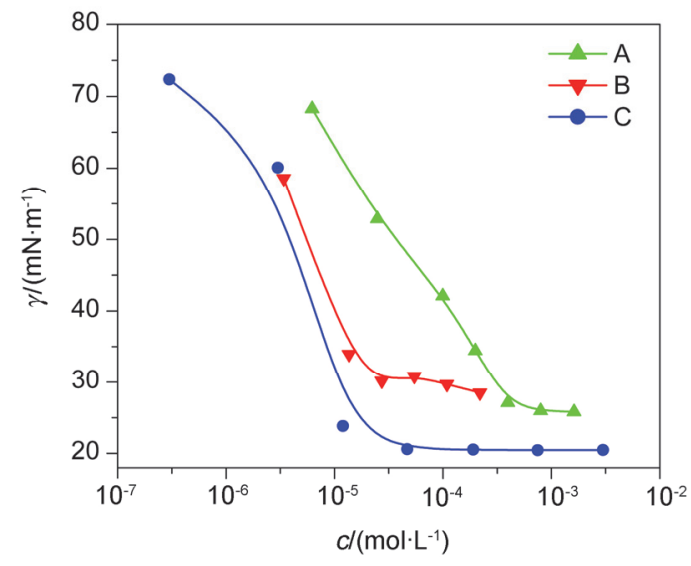

图 1 A $(\mathbf{8}: \mathbf{9}=59 \%: 41 \%) 、 \mathrm{~B}(\mathbf{1 0}: \mathbf{1 1}=67 \%: 33 \%)$ 和 C $(\mathbf{6}: \mathbf{7}=$ $65 \%: 35 \%)$ 的表面张力随浓度变化曲线

Figure 1 Surface tension values of A $(\mathbf{8}: \mathbf{9}=59 \%: 41 \%)$, B $(\mathbf{1 0}: \mathbf{1 1}$ $=67 \%: 33 \%)$ and $\mathrm{C}(6: 7=65 \%: 35 \%)$

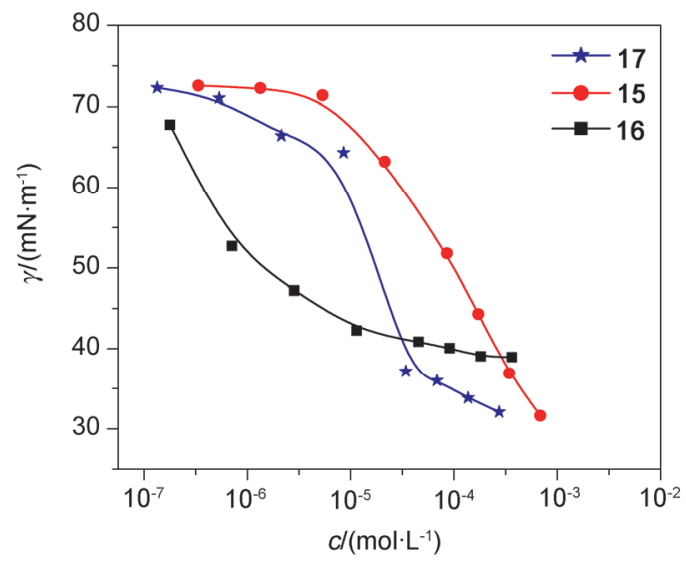

图 2 化合物 15、16 和 17 的表面张力随浓度变化曲线 Figure 2 Surface tension values of $\mathbf{1 5}, \mathbf{1 6}$ and $\mathbf{1 7}$ 


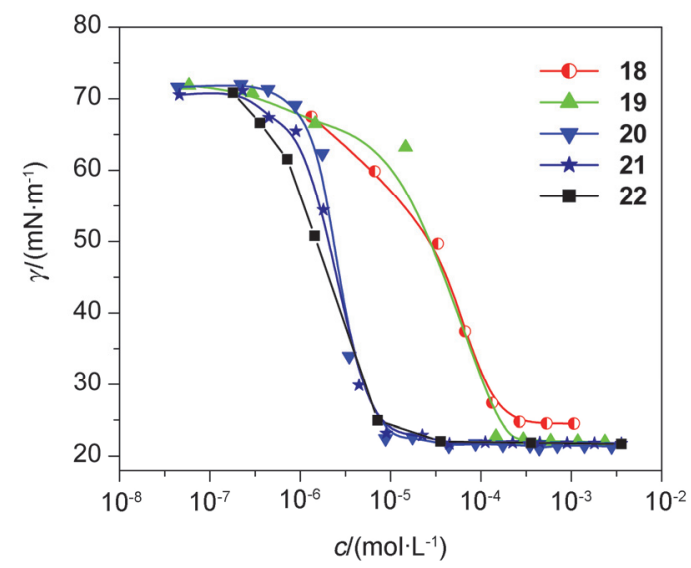

图 3 化合物 $18 \sim 22$ 的表面张力随浓度变化曲线

Figure 3 Surface tension values of $\mathbf{1 8} \sim \mathbf{2 2}$

浓度下的表面张力值为 $22.7 \mathrm{mN} / \mathrm{m}$; 化合物 $\mathbf{2 0}$ 的临界胶 束浓度为 $4.4 \times 10^{-5} \mathrm{~mol} / \mathrm{L}$, 临界胶束浓度下的表面张力 值为 $21.4 \mathrm{mN} / \mathrm{m}$; 化合物 21 的临界胶束浓度为 $4.5 \times$ $10^{-5} \mathrm{~mol} / \mathrm{L}$, 临界胶束浓度下的表面张力值为 21.7 $\mathrm{mN} / \mathrm{m}$; 化合物 22 的临界胶束浓度为 $4.6 \times 10^{-5} \mathrm{~mol} / \mathrm{L}$, 临界胶束浓度下的表面张力值为 $21.9 \mathrm{mN} / \mathrm{m}$. 结果表明, 化合物 $20 \sim 22$ 降低水溶液表面张力的能力强、效率高, 它们的表面活性性能均优于全氟辛酸钠(临界胶束浓度 为 $3.1 \times 10^{-2} \mathrm{~mol} / \mathrm{L}$, 临界胶束浓度下的表面张力值为 $24.7 \mathrm{mN} / \mathrm{m}^{[32]}$ ), 可作为潜在的 PFOS/PFOA 替代物. 表 面张力测试结果还表明, 聚合度 $n$ 的值等于或大于 4 后, 化合物降低水溶液表面张力的能力和效率变化很小.

综上，表面活性剂在水溶液中的溶解度对表面活性 性能影响较大. 考虑到磺酸基团的亲水性很强, 我们设 计并成功合成了亲水基为磺酸基团的氟表面活性剂 23 . 化合物 23 可经两步反应以 75\%的总收率得到, 正如所 料, 其水溶性较大, 其水溶液的表面张力随浓度变化的 测试结果如图 4, 此外, 也对其在不同浓度 $(1.0 \mathrm{~g} / \mathrm{L}$ 和 5.0 $\mathrm{g} / \mathrm{L}$ )氯化钠水溶液下的活性进行了研究. 由图 4 知, 化 合物 23 的临界胶束浓度为 $9.9 \times 10^{-3} \mathrm{~mol} / \mathrm{L}$, 临界胶束 浓度下的表面张力值为 $21.3 \mathrm{mN} / \mathrm{m}$, 其活性也优于全氟 辛酸钠, 且其在盐溶液中的表面张力值更低些说明其具 有很好的抗盐性.

由 15 与 $\mathrm{A}$ (图 5)、 17 与 $\mathrm{B} 、 20$ 与 $\mathrm{C}$ 的性能对比知, 苯 环上亲水基的位置对化合物的溶解度以及表面活性性 能有一定的影响 (因为甲基的空间位阻较小, 此处忽略 甲基的影响). 亲水基在苯环上对位的表面活性剂溶解 度大于亲水基在间位上的表面活性剂. 化合物溶解度增 大, 水溶液最低表面张力值明显降低, 但表面张力值随 浓度变化的趋势一致。

由化合物 15、16、17、20 和 23 的性能对比(图 6) 知, 亲水基结构对化合物的溶解度以及表面活性性能有 一定的影响. 亲水基为磺酸基团的化合物 23 降低水溶 液表面张力的能力最强, 但是效率低; 亲水基为四聚乙

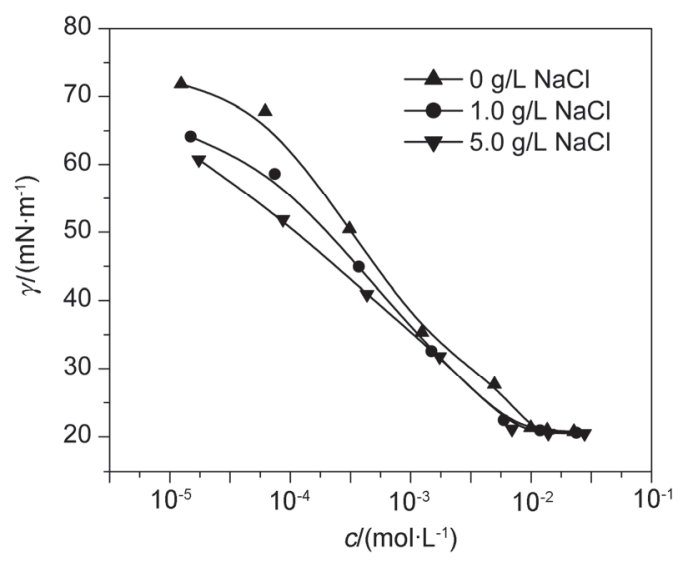

图 4 化合物 23 在水或 $\mathrm{NaCl}$ 水溶液中的表面张力随浓度变化曲线 Figure 4 Effect of electrolyte on surface tension of $\mathbf{2 3}$

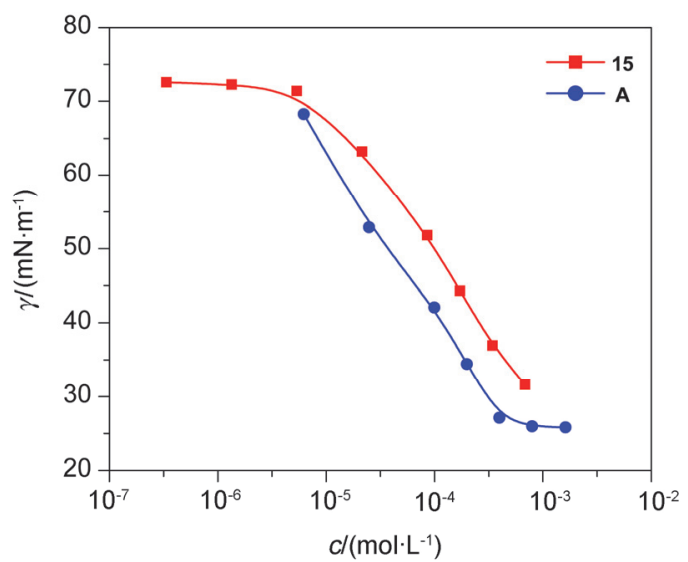

图 515 与 $\mathrm{A}$ 的表面张力随浓度变化曲线

Figure 5 Surface tension values of $\mathbf{1 5}$ and A

二醇的化合物 20 降低水溶液表面张力的能力次之, 效 率最高; 化合物 23 的溶解度比化合物 $15 \sim 17$ 的溶解度 大将近 100 倍; 化合物 15 17 的溶解度非常低, 它们的 饱和溶液表面张力值仍旧很高, 表面活性性能较差.

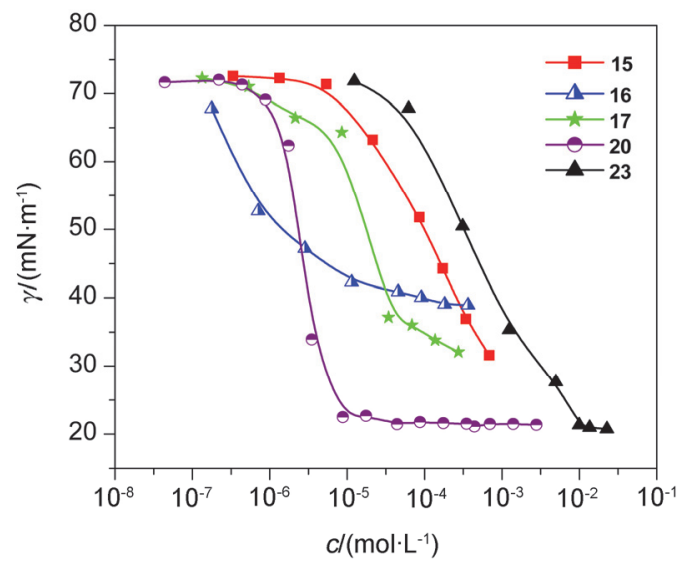

图 6 化合物 15、16、17、20 和 23 的表面张力随浓度变化曲线 Figure 6 Surface tension values of 15、16、17、20 and 23 


\section{3 急性毒性结果与讨论}

我们选择最具应用前景的化合物 20 作为检测样品, 合成了 $150 \mathrm{~g}$ 样品委托上海化工研究院检测中心对其急 性经口毒性进行测试. 实验用鼠为 ICR 小鼠(清洁级), 购买于上海市杰思捷实验动物有限公司, 动物给药之前 禁食一夜. 每个剂量下用 10 只小鼠(5 只雄性和 5 只雌 性), 四个剂量分别为 $1000 、 2150 、 4640$ 和 $10000 \mathrm{mg} / \mathrm{kg}$. 测试环境符合 GB 14925 的要求, 测试方法和标准依据 GB 21603. 根据测试结果, 在当前测试条件下, 试验样 品急性口服毒性 $\mathrm{LD}_{50}$ (半数致死量)值为 $3160 \mathrm{mg} / \mathrm{kg}$, 比 全氟辛酸铵的值 $\left(\mathrm{C}_{7} \mathrm{~F}_{15} \mathrm{COONH}_{4}, 470 \sim 540 \mathrm{mg} / \mathrm{kg}\right)$ 高, 说 明其毒性低于全氟辛酸铵, 为低毒.

用理论与计算化学方法预测化合物 1-1 和 1-2 的急 性毒性, 结果见表 1 . 由表 1 知, 化合物 1-1 和 1-2 的急 性毒性接近, 均为低毒.

急性毒性测试和急性毒性预测结果均表明，基于 HFPD 合成的氟表面活性剂为低毒.

表 1 化合物 1-1 和 1-2 的急性毒性预测结果

Table 1 Acute toxicity of compounds 1-1 and 1-2

\begin{tabular}{ll}
\hline Surfactants & Acute toxicity $^{a}$ \\
\hline $\mathbf{1 - 1}$ & 4.43 \\
$\mathbf{1 - 2}$ & 4.40 \\
\hline
\end{tabular}

1-1.999: rat, oral, $\mathrm{LD}_{50}<1 \mathrm{mg} / \mathrm{kg} ; 2-2.999$ : rat, oral, $\mathrm{LD}_{50} 1 \sim 49 \mathrm{mg} / \mathrm{kg}$; 3-3.999: rat, oral, $\mathrm{LD}_{50} 50 \sim 499 \mathrm{mg} / \mathrm{kg}$; 4-4.999: rat, oral, $\mathrm{LD}_{50} 500 \sim 4999$ $\mathrm{mg} / \mathrm{kg} ;>5$ : rat, oral, $\mathrm{LD}_{50}>5000 \mathrm{mg} / \mathrm{kg}$.

\section{3 结论}

本文设计并合成了含全氟支链的阳离子、双子和两 性型表面活性剂, 它们的水溶性均不好, 表面活性性能 也都不太理想. 设计合成了一系列不同长度亲水链的含 全氟支链的非离子型表面活性剂, 随着聚合度 $n$ 值的增 加水溶性增大, 表面活性性能增强, 但是, $n$ 等于或大于 4 后, 性能变化不明显. 其中, 化合物 $20 \sim 22$ 因具有很 好的性能可作为潜在的替代物. 化合物 13 合成简单易 于实现工业化生产, 可作为开发新型氟表面活性剂的理 想中间体. 化合物 23 可经两步反应得到, 合成操作简 单、条件温和、成本低、产率高. 该化合物降低水溶液 表面张力的能力强, 可将水溶液表面张力降低至 20.8 $\mathrm{mN} / \mathrm{m}$, 其还具有很好的抗盐性, 可作为 PFOA 的潜在替 代物.

急性毒性测试结果表明，化合物 20、1-1 和 1-2 为 低毒, 毒性均低于全氟辛酸铵. 此外, 这些化合物总的 氟碳数少(6 个), 它们的降解性应该比含全氟辛基的化 合物好. 因此, $\mathrm{CF}_{3} \mathrm{CF}_{2} \mathrm{CF}_{2} \mathrm{C}\left(\mathrm{CF}_{3}\right)_{2}$-基团可作为理想的氟 表面活性剂的疏水链.

综上，我们认为基于 HFPD 中碳碳双键反应位点合 成支链型氟表面活性剂是一种简单、环保、经济的研发 PFOA/PFOS 替代品的方法. 有关这些化合物的降解性
研究正在进行中.

\section{4 实验部分}

\section{1 仪器与试剂}

全氟-2-甲基-2-戊烯从宿迁凯尔氟有限公司购买, 纯度为 $99 \%$. 其它试剂均为分析纯试剂, 未经提纯, 直 接使用. 溶剂均为分析纯, 需要除水的溶剂均按照标准 操作严格除水后加入 $4 \AA$ 分子篮干燥备用. 柱层析用硅 胶为烟台江友硅胶开发有限公司生产的硅胶 $\mathrm{H}($ 颗粒度： $10 \sim 40 \mu)$. 核磁共振氢谱和氟谱由 Varian Mercuryvx300 测定，核磁共振碳谱由 Varian Mercury-400 测定， 红外光谱经 Nicolet 380FT-IR 测定. EI 低分辨质谱由 Agilent 5973N 型质谱仪测定, ESI 低分辨质谱由 Agilent 1200/G6100A 型质谱仪测定，MALDI 低分辨质谱由 AXIMA Performance MALDI-TOF/TOF 型质谱仪测定， EI 高分辨质谱由 Waters Micromass GTC-CA176 型质谱 仪测定, ESI 高分辨质谱由 Thermo Fisher Scientific LTQ FT Ultra 型质谱仪测定, MALDI 高分辨质谱由 IonSpec 4.7 Tesla FT 型质谱仪测定.

\section{2 合成方法}

所有化合物的合成方法、实验步骤、表征数据及部 分化合物的原始谱图见支持信息.

\section{References}

[1] Song, A.-X.; Dong, S.-L.; Hao, J.-C.; Liu, W.-M.; Xu, G.-Y.; Wang, H.-Q. J. Fluorine Chem. 2005, 126, 1266.

[2] Lu, C. Q.; Kim, J. H.; DesMarteau, D. D. J. Fluorine Chem. 2010, $131,17$.

[3] Gramstad, T. R.; Haszeldine, N. J. Chem. Soc. 1957, 4069.

[4] Krafft, M. P.; Riess, J. G. J. Polym. Sci., Part A: Polym. Chem. 2007, 45,1185 .

[5] Kaplánek, R.; Paleta, O.; Ferjentsiková, I.; Kodícek, M. J. Fluorine Chem. 2009, 130, 308.

[6] Caillier, L.; Taffin de Givenchy, E.; Levy, R.; Vandenberghe, Y.; Geribaldi, S.; Guittard, F. J. Colloid Interface Sci. 2009, 332, 201.

[7] Prescher, D.; Gross, U.; Wotzka, J.; Txchen-Schlueter, M.; Starke, W. Acta Hydrochim. Hydrobiol. 1985, 13, 17.

[8] Key, B. D.; Howell, R. D.; Criddle, C. S. Environ. Sci. Technol. 1997, 31, 2445.

[9] Key, B. D.; Howell, R. D.; Criddle, C. S. Environ. Sci. Technol. 1998, 32, 2283.

[10] Houde, M.; Martin, J. W.; Letcher, R. J.; Solomon, K. R.; Muir, D. C. G. Environ. Sci. Technol. 2006, 40, 3463.

[11] U.S. Environmental Protection Agency, http://www.epa.gov/ oppt/pfoa/pubs/stewardship/index.html.

[12] Drakesmith, F. G.; Hughes, D. A. J. Appl. Electrochem. 1979, 9, 685.

[13] Haszeldine, R. N. Nature 1951, 167, 139.

[14] Haszeldine, R. N. J. Chem. Soc. 1949, 2856.

[15] Murphy, P. M.; Baldwin, C. S.; Buck, R. C. J. Fluorine Chem. 2012 138,3 .

[16] Boutevin, G.; Tiffes, D.; Loubat, C. J. Fluorine Chem. 2012, 134, 7.

[17] Dmowski, W.; Plenkiewicz, H.; Piasecka-Maciejewska, K.; Prescher, D.; Schulze, J.; Endler, I. J. Fluorine Chem. 1990, 48, 77.

[18] Liu, Z.-M.; Wu, J.-F.; Tan, L.-M.; Hu, Y.-M.; Song, Z.-C.; Yu, X.-X. Fine Chem. 2005, 22, 53. (刘在美, 吴京峰, 谈龙妹, 胡应 模, 宋志超, 俞雪兴, 精细化工, 2005, 22, 53.)

[19] Yang, B.-Q.; Wang, L.; Yang, J.; Liu, J. Chem. Res. Appl. 2013, 25 , 1523. (杨百勤, 主林, 杨健, 刘瑾, 化学研究与应用, 2013, 25, 1523.)

[20] Zheng, S.-C.; Zhao, Y.-J.; Lei, Z.-G.; Zhou, Q.; Wang, S.-H. Chem. 
Prod. Technol. 2012, 19, 6. (郑士才, 赵颖俊, 雷志刚, 周强, 王树 华，化工生产与技术, 2012, 19, 6.)

[21] Qi, H.; Xu, W.-G.; Zhao, W.-J.; Chen, W.; Liu, Y.-L.; Zhang, Y.-Y. Organo-Fluorine Industry 2012, 4, 18. (齐海, 徐卫国, 赵卫娟, 陈 伟，刘毓林，张勇耀，有机氟化工， 2012, 4, 18.)

[22] Xu, J.-H.; Chen, Z.-J.; Zhang, W.-B. Organo-Fluorine Industry 2007, 3, 29. (徐金和, 陈志军, 张巍彪，有机氟化工, 2007, 3, 29.)

[23] Ikeda, I.; Tsuji, M.; Okahara, M. Tenside, Surfactants, Detergents 1987, 24, 272 .

[24] Tomota, H.; Nakayama, N. JP 03093744, 1991 [Chem. Abstr. 1991, $115,231866]$.

[25] Koga, K.; Nemoto, F. JP 61069754, 1986 [Chem. Abstr. 1986, 105, 133514].

[26] Zhou, H.-T.; Gao, A.-T.; Xing, H.; Gou, Z.-M.; Xiao, J.-X. Acta Chim. Sinica 2011, 69, 1035. (周洪涛, 高岸涛, 邢航, 勾志明, 肖 进新, 化学学报, 2011, 69, 1035.)
[27] Wang, C.; Chen, X.-Y.; Zhu, Z.; Xiao, J.-X. Acta Chim. Sinica 2009, 67, 1425. (王晨, 陈新远, 朱湛, 肖进新, 化学学报, 2009, 67, 1425.)

[28] Xing, H.; Lin, C.-X.; Xiao, J.-X. Acta Chim. Sinica 2008, 66, 1382. (邢航, 林崇熙, 肖进新, 化学学报, 2008, 66, 1382.)

[29] Jin, C.; Yan, P.; Wang, C.; Xiao, J.-X. Acta Chim. Sinica 2005, 63, 279. (金辰，严鹏，王晨，肖进新，化学学报, 2005, 63, 279.)

[30] Sha, M.; Pan, R.-M.; Zhan, L.-W.; Xing, P.; Jiang, B. Chin. J. Chem. 2014, 32, 995.

[31] Sha, M.; Pan, R.-M.; Xing, P.; Jiang, B. J. Fluorine Chem. 2015, $169,61$.

[32] Zhang, Q.-S.; Luo, Z.-Y.; Curran, D. P. J. Org. Chem. 2000, 65, 8866.

(Cheng, F.) 